\title{
Herschel SPIRE FTS spectral line source calibrators
}

Hopwood, Rosalind; Polehampton, Edward; Valtchanov, Ivan; Fulton, Trevor; Lu, Nanyao; Marchili, Nicola; Naylor, David; Makiwa, Gibion; Spencer, Locke; Swinyard, Bruce; van der Wiel, Matthijs H D

Published in:

Proceedings Fourier Transform Spectroscopy and Hyperspectral Imaging and Sounding of the Environment

DOI:

10.1364/FTS.2015.FT3A.5

Publication date:

2015

Document version

Publisher's PDF, also known as Version of record

Citation for published version (APA):

Hopwood, R., Polehampton, E., Valtchanov, I., Fulton, T., Lu, N., Marchili, N., Naylor, D., Makiwa, G., Spencer, L., Swinyard, B., \& van der Wiel, M. H. D. (2015). Herschel SPIRE FTS spectral line source calibrators. In Proceedings Fourier Transform Spectroscopy and Hyperspectral Imaging and Sounding of the Environment The Optical Society (OSA). https://doi.org/10.1364/FTS.2015.FT3A.5 


\title{
Herschel SPIRE FTS Spectral Line Source Calibrators
}

\author{
Rosalind Hopwood ${ }^{1}$, Edward Polehampton ${ }^{2,3}$, Ivan Valtchanov ${ }^{4}$, Trevor Fulton ${ }^{3}$, \\ Nanyao $\mathrm{Lu}^{5}$, Nicola Marchili ${ }^{6}$, David Naylor ${ }^{3}$, Gibion Makiwa ${ }^{3}$, Locke Spencer ${ }^{3}$, Bruce \\ Swinyard $^{2,7}$, and Matthijs van der Wiel ${ }^{3,8,9}$ \\ ${ }^{1}$ Department of Physics, Imperial College London, Prince Consort Road, London SW7 2AZ, UK \\ ${ }^{2}$ RAL Space, Rutherford Appleton Laboratory, Chilton, Didcot, Oxfordshire, OX11 OQX, UK \\ ${ }^{3}$ Institute for Space Imaging Science, Department of Physics \& Astronomy, University of Lethbridge, 4401 University \\ Drive, Lethbridge, Alberta, T1K 3M4, Canada \\ ${ }^{4}$ Herschel Science Centre, European Space Astronomy Centre, ESA, 28691 Villanueva de la Cañada, Spain \\ ${ }^{5}$ NASA Herschel Science Center, MS 100-22, California Institute of Technology, Pasadena, CA 91125, USA \\ ${ }^{6}$ Università di Padova, I-35131 Padova, Italy \\ ${ }^{7}$ Department of Physics and Astronomy, University College London, Gower St, London WC1E 6BT, UK \\ ${ }^{8}$ Niels Bohr Institute, Copenhagen University, Juliane Maries Vej 30, DK-2100 Copenhagen Ø, Denmark \\ ${ }^{9}$ Centre for Star and Planet Formation, Natural History Museum of Denmark, University of Copenhagen, Øster \\ Voldgade 5-7, DK-1350 Copenhagen K, Denmark \\ r.hopwood@imperial.ac.uk
}

\begin{abstract}
We present a summary of the Herschel SPIRE/FTS calibration programme to monitor the repeatability of spectral lines. Observations of planetary nebulae and post-AGB stars are used to assess repeatability and model the asymmetry of the instrument line shape.
\end{abstract}

OCIS codes: $350.1270,300.6270,300.6300$.

\section{Introduction}

The ESA Herschel Space Observatory [1] operated over a period of four years, from May 2009-April 2013, and made observations of the far infrared and sub-millimetre sky. The Spectral and Photometric REceiver (SPIRE) [2] is one of three focal plane instruments on board Herschel, and consists of an imaging photometric camera and an imaging Fourier Transform Spectrometer (FTS). The FTS provided simultaneous frequency coverage in the sub-millimetre with two bolometer arrays: SLW $(447-1018 \mathrm{GHz})$ and SSW $(944-1568 \mathrm{GHz})$. The basic calibration scheme and the calibration accuracy for the FTS is described in [3] and a detailed analysis of the routine-phase calibration sources is presented in [4]. This paper summarises some of the interesting issues and results from the calibration programme.

\section{FTS routine-phase calibration programme}

A programme covering a range of targets was devised before launch in order to provide calibration and monitor the performance of the Herschel SPIRE FTS. This programme included observations of a region of dark sky (for extended calibration), observations of planets (including Uranus to derive the point-source calibration), and observations of asteroids and line sources (evolved stars). Sources were chosen to provide continuous coverage and to maximise ancillary data from other telescopes. The spectral line sources were observed 21-31 times through the mission at high resolution $(-0.555-12.645 \mathrm{~cm})$ and up to 11 times at low resolution $(-0.555-0.560 \mathrm{~cm})$. See [4] for a detailed summary of these observations.

\section{Data processing}

Data processing for Herschel is carried out with a dedicated package, the Herschel Interactive Processing Environment (HIPE; [5]), which allows Jython scripting and is based on tasks written in JAVA. The SPIRE FTS pipeline processing is carried out in three stages [6]: the time domain (e.g. deglitching, linearisation of bolometer signals, correction for detector saturation); the spatial domain (e.g. interferogram baseline removal, 2nd level deglitching, phase correction); and the spectral domain (e.g. correction for instrument and telescope emission, calibration, averaging). The standard point source calibration is based on observations and models of the planet Uranus. Figure 1 [3] shows a typical FTS interferogram and the corresponding spectrum. Further interactive processing may be necessary for sources embedded in an astronomical background, where the background is removed using the off-axis bolometers [7]. 
The main routine calibration sources were repeatedly observed throughout the mission, allowing a relative correction for pointing uncertainty to be determined by comparing different measurements [8]. The pointing corrected data provide a comparison set for the standard point-source processing.
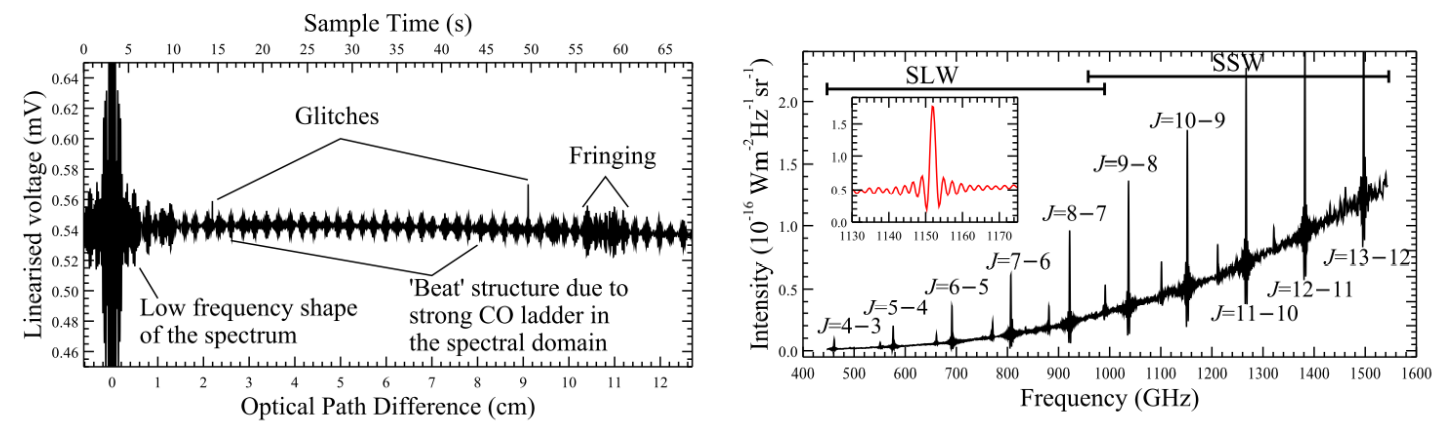

Fig. 1. Left: a typical FTS interferogram for a source with strong ${ }^{12} \mathrm{CO}$ lines, illustrating the appearance of fringing and two strong glitches. Right: the final spectrum corresponding to the interferogram, showing the SLW and SSW bands. A zoom of the ${ }^{12}$ CO $J=10-9$ line is shown in the insert. The glitches (e.g. cosmic ray impacts) are addressed in the standard pipeline, and the fringing is removed by the calibration, for all but the brightest sources (e.g. brighter than Uranus).

\section{Line Source Repeatability}

\subsection{Line fitting}

Spectral line fitting was applied to the spectra for each line source, using a simultaneous fit of sinc profiles for the spectral lines $[9,10]$ and a polynomial of order 3 for the continuum [4]. Figure 2 shows an example fit for the source NGC7027. The strongest lines in the spectrum are due to ${ }^{12} \mathrm{CO}$ rotational transitions, and the best fit sinc profiles for these lines were used for the further analysis. Line position and integrated line flux were extracted from the sinc parameters. The spectral line signal-to-noise ratio (SNR) was also measured using the fitted peak and the local noise in the residual spectrum, after subtracting the total fitted model.
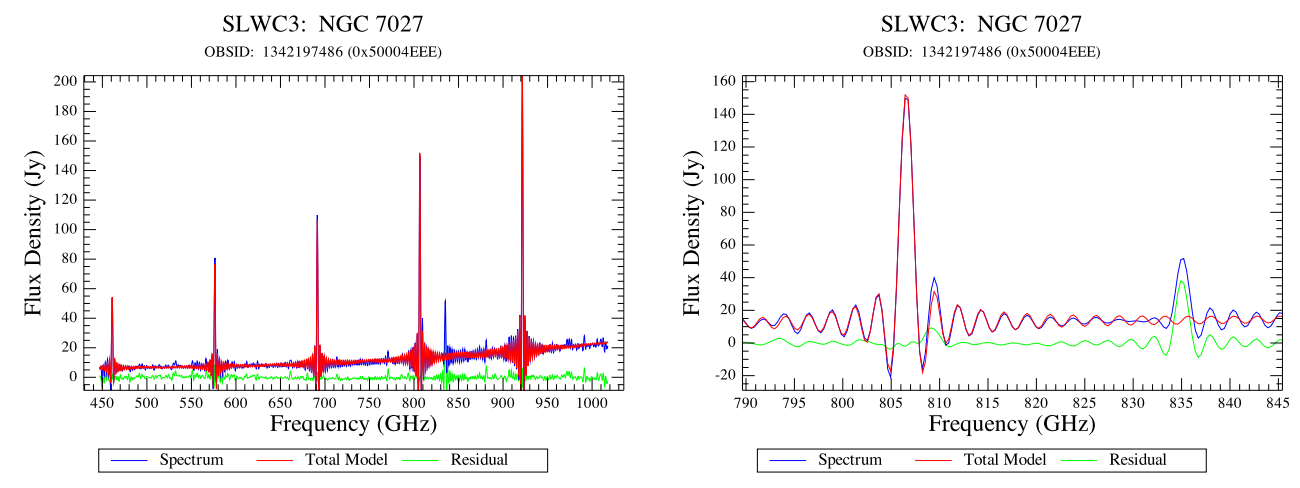

Fig. 2. An illustration of the fitting process applied to the FTS spectra. Here only the ${ }^{12} \mathrm{CO}$ lines are fitted to highlight the need to include all significant features to be fitted simultaneously. The left panel shows the full spectrum. The right panel shows a zoom around the ${ }^{12} \mathrm{CO} J=7-6$ line. The asymmetry discussed in the Line Shape section has a clear influence on the residual. 


\subsection{Results}

The repeatability of the SPIRE FTS was assessed using the relative spread in integrated line flux and the spread of line position. Figure 3 shows a repeatability of better than $6 \%$. For sources where there are multiple observations available, it is possible to correct the data for pointing offset [8], in which case the repeatability improves to $1-2 \%$. The spread on fitted line position is better than $7 \mathrm{~km} \mathrm{~s}^{-1}$, which is $\sim 1-3 \%$ of the resolution element.
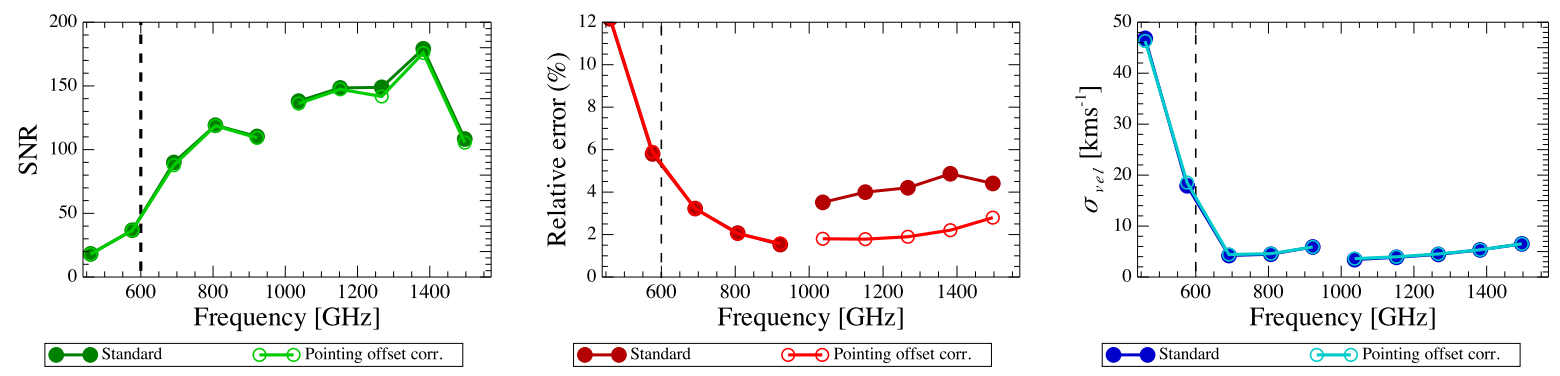

Fig. 3. SNR (left), relative error on the integrated line flux (middle) and spread in measured velocity (right). For lines above $600 \mathrm{GHz}$, with a SNR $>50$, the repeatability is better than $6 \%$ and better than $7 \mathrm{~km} \mathrm{~s}^{-1}$. Correcting for pointing offset improves the repeatability of line flux, but does not impact the measured position.

\section{Line Shape}

In order to assess any difference between a pure sinc and the actual instrument line shape, the ${ }^{12} \mathrm{CO}$ lines in the NGC7027 spectrum were used. These lines have high signal-to-noise ( $>200)$, and suffer little blending from neighbouring spectral lines. The data from 31 high resolution observations were combined. The continuum and all other weaker lines were fitted and subtracted, leaving only the strong ${ }^{12} \mathrm{CO}$ lines. These lines were then cut-out, normalised, re-centred and averaged to produce an empirical line profile. Fitting this empirical profile with a sinc function shows there is a slight asymmetry to the actual instrument line shape, most prominent for the first side lobe on the higher frequency side. By integrating over the fitted sinc and the empirical profile, a difference of $2.6 \%$ was found, which is the systematic error on any extracted line flux. This asymmetry may be due to a residual phase shift in the interferogram at high path difference, and thus it may be possible to correct in future version of HIPE.

\section{Conclusions}

Observations of the routine-phase calibration line sources show the SPIRE FTS to be highly stable throughout the Herschel mission, with a repeatability of better than $6 \%$ for lines with SNR $>50$. A slight asymmetry in the instrumental line shape introduces a systematic error of $2.6 \%$ on the measure integrated line flux.

\section{References}

1. Pilbratt, G. L., Riedinger, J. R., Passvogel, T. et al., A\&A 518, L1 (2010)

2. Griffin, M. J., Abergel, A., Abreu, A. et al., A\&A 518, L3 (2010)

3. Swinyard, B. M., Polehampton, E. T., Hopwood, R., et al., MNRAS 440, 3658 (2014)

4. Hopwood, R., Polehampton, E. T., Valtchanov, I.,et al., in preparation

5. Ott, S., ADASS XIX, edited by Y. Mizumoto, K.-I. Morita, \& M. Ohishi, ASP Conf. Series, 434139 (2010)

6. Fulton, T., Polehampton, E. T., Valtchanov, I.,et al., in preparation

7. Polehampton, E. T., et al., Astronomical Society of the Pacific Conference Series Vol. 0, ADASS XXIV, in press

8. Valtchanov, I., Hopwood, R., Polehampton, E. T., et al., Experimental Astronomy 37, 207 (2014)

9. Spencer, L. D., Naylor D. A., \& Swinyard, B. M., Meas. Sci. Technol. 21, 065601 (2010)

10. Naylor, D. A., Baluteau, J.-P., Barlow, M. J., et al., Society of Photo-Optical Instrumentation Engineers (SPIE) Conference Series Vol. 7731 